\title{
Editorials
}

\section{Writing for journals: a paradigm lost?}

Writing for journals, like giving to the poor and taking exercise, is one of those institutions that seem beyond reproach. One of the encouraging characteristics of our civilisation (as the ideology has it) is this constant stream of high quality information about our bodies and our health, contributing piece by piece to an expanding knowledge base, and taking us on the long slow march to a brighter and healthier future.

The evidence for these assumptions is remarkably thin, which is somewhat surprising in view of the fact that they are usually expounded by a profession that prides itself on its objectivity. The assumption that publication is the same as dissemination has never been satisfactorily tested; indeed one study found that those who claimed to have read journals recently had retained surprisingly little of the information contained in them. ${ }^{1}$

This does not surprise professional communicators ${ }^{2}$ because most scientific papers break the principles of effective style, famously outlined by George Orwell, such as short sentences, short and familiar words and active voice. ${ }^{3}$ They ignore the popular technique, well validated by the research and experiences of publishing houses, of starting with an explicit key message; instead they favour the tortuous four section structure (also known as the IMRADIntroduction, Methods, Results and Discussionstructure) where a clear message will appear in the last sentence in just under $50 \%$ of articles. ${ }^{4}$ The text in scientific journals is neatly laid out, but grey and uninviting, without attention drawing devices such as photographs and illustrations, cross heads and pull out quotes, and headlines with verbs (though there are signs that this may be changing ${ }^{5}$ ).

But, defendants say, style and readability are just a minor part of the process. What really matters is the excellence of the content. and this is assured in scientific and medical publishing by peer review, a feature that makes this form of publishing more valuable than other. Again, this assumption is rarely tested. Peer review is based on a dubious premise, which is that the best way to improve quality is to ask the opinion of rivals. The system may be good at identifying problems of detail, but is notoriously bad at spotting fraud. ${ }^{6}$ Perhaps most important, reviewing is advisory only, and editors reserve and exercise the right to fly in the face of this advice.

A more disturbing concern stems from the motivation of those who write scientific papers. The accepted ideology is that they are written by committed professionals to communicate interesting advances. But that is probably not true: from my experiences running several hundred courses for public health writers over the past 10 years, clearly for many, if not most, the main reason is one of obligation - specifically that failure to publish will seriously harm an individual's career. Reluctance and obligation are hardly sound planks on which a profession should build its knowledge base.

Of course, this may not matter: if a professional group wishes to put pressure on members to publish, then that is its prerogative. But there is an argument that public health could and should be different. As its title suggests, it sets out to be "public", and its own definition of its role is "the science and art of preventing diseases, prolonging life and promoting health through organised efforts of society" ${ }^{7}$ In this context, the current emphasis on writing scientific papers may cause two main problems.

The first is that it distorts the writing culture. An effective use of written language is one of the main therapeutic tools in public health, but there is constant pressure to mould non-academic types of writing, such as reports, press releases and patient information, into the form and tone of a scientific paper. This can lead to inaccurate reports, as with the World Health Organisation's MONICA project (MONItoring CArdiovascular diseases) ${ }^{8}$ In what has been called the Pulse Paradox, ${ }^{9}$ doctors accord high status to academic journals, and low status to medical newspapers, which they call the funnies or comics, even though they read them. This helps to ensure that the techniques these media use remain generally outside public health.

There is also the question of resources. Writing a scientific paper is a time consuming activity. Assuming, at a conservative estimate, a total of one week of writing time (from all coauthors) per paper, then this journal alone takes up the services of two full time public health doctors a year-at no cost to the journal. Is this a justifiable use of public money? The dilemma is particularly acute in developing countries: should public health doctors invest time writing papers for international journals, or should they devote their scarce time and resources to local health issues? Newsletters and e-mails provide cheaper and usually more effective alternatives, so the question of the need to communicate is no longer an issue.

It would be a major gain if we could reduce the general obligation on public health professionals to write for publication. A truly radical approach would be for each professor of public health to limit the output from each department to two papers a year, chosen through internal competition; the remainder could be posted on the department's web site. At a stroke we would raise the standard of the literature, and (by reducing writing and reading time) release time for other activities. It may push bureaucratic procedures based on publication (for example the United Kingdom universities' research assessment exercise) into complete disarray, but as everyone complains about the unfairness of the system, this would be a great advance.

Other measures are perhaps more easily achieved. The editors of this journal can take the lead by reducing drastically the space available to publishing scientific papers; they could then increase drastically the space available to well presented reviews (written in the style of good magazine articles, rather than turgid scientific papers or editorials) to disseminate new findings and good practice. The teaching of real written communication skills can replace the teaching of assembling scientific papers. Critical appraisal skills could increase to take account not only of the content of a scientific paper, but also its potential value as communication. Public health practitioners would be the appropriate group to lead the way towards a radical rethink of the role of scientific papers and to set a new agenda for writing for journals

I suspect the changes would be hard to affect, mainly because those who have mastered the techniques of writing 
papers are those who are running most of our institutions. But, as the editors of this journal have written: "The destiny of many specialised journals is to wait in the shelves of a library until a researcher looks for a particular page on a specialised topic. We believe in products designed for the use of individuals and not just by institutions." 10 Where better to start than by taking away the obligation that public health doctors should spend so much time writing, unwillingly, for journals that are scarcely read.

TIM ALBERT

Tim Albert Training, Paper Mews Court, 284 High Street, Dorking, Surrey RH4 1QT
1 Kellett CF, HartAL, Price CJ, et al. Poor recall performance of journal borrowing doctors. Lancet 1996;348:479.

2 O' Donnell M. Evidence-based illiteracy: time to rescue 'the literature'. Lancet 2000;355:488-90.

3 Orwell G. Politics and the English language. In: Inside the whale and other essays. London: Penguin Books, 1957.

4 Albert T. Winning the publications game. 2nd ed. Abingdon: Radcliffe Medical Press, 2000:41.

5 Goodman, NW. Survey of active verbs in the titles of clinical trial reports. BMҰ 2000;320:914-15.

6 Horton R. After Bezwoda. Lancet 2000;355:942-3.

7 Anon. What is public health. Web site of Faculty of Public Health Medicine, London (as at 26 March 2000)

8 Tunstall-Pedoe H. Did MONICA really say that? BMF 1998;317:1023.

9 Albert T. $A-Z$ of medical writing. London: BMJ Books, 2000:106.

10 Alvarez-Dardet C, Ashton JR. And now-the next 50 years! A new editorial team takes over. $\mathcal{F}$ Epidemiol Community Health 1998:52:1. 